\title{
Search for neutrino bursts from core collapse supernovae at the Baksan Underground Scintillation Telescope.
}

\author{
R.V. Novoseltseva, M.M. Boliev, I.M. Dzaparova, M.M. Kochkarov, S.P. Mikheyev, \\ Yu.F. Novoseltsev, V.B. Petkov, P.S. Striganov, G.V. Volchenko, V.I. Volchenko, A.F. Yanin* \\ *Institute for Nuclear Researsh of the Russian Academy of Sciences, \\ 60-th October Anniversary prospect 7a, Moscow 117312, Russia
}

\begin{abstract}
Current status and results of the experiment on recording neutrino bursts are presented. The observation time (since 1980) is 24.7 years. The upper bound of collapse frequency in our Galaxy is $0.093 y^{-1}(\mathbf{9 0 \%}$ CL).
\end{abstract}

Keywords: neutrino, supernova, galactic sources

\section{INTRODUCTION}

One of the current task of the Baksan Underground Scintillation Telescope (BUST) is the search for neutrino bursts from gravitational collapse of stars.

BUST is located in the Northern Caucasus in the underground laboratory at the effective depth of $8.5 \times$ $10^{4} \mathrm{~g} \cdot \mathrm{cm}^{-2}(850 \mathrm{~m}$ of w.e.) [1]. The facility has dimensions $17 \times 17 \times 11 \mathrm{~m}^{3}$ and consists of four horizontal scintillation planes and four vertical ones (Fig. 1). Five planes of them are external planes and three lower horizontal planes are internal ones. The upper horizontal plane consists of $576(24 \times 24)$ liquid scintillator detectors of the standard type, three lower planes have $400(20 \times 20)$ detectors each. The vertical planes have $15 \times 24$ and $15 \times 22$ detectors. The detector sizes are $0.7 \times 0.7 \times 0.3 \mathrm{~m}^{3}$. The distance between neighboring horizontal scintillation layers is $3.6 \mathrm{~m}$. The angular resolution of the facility is $2^{\circ}$, time resolution is $5 \mathrm{~ns}$.

The information from each detector is transmitted over three channels: an anode channel (which serves for trigger formation and amplitude measurements up to $2.5 \mathrm{GeV}$ ), a pulse channel with operation threshold $12 \mathrm{MeV}$ (since 1991 this threshold $=8 \mathrm{MeV}$; the most probable energy deposition of a muon in a detector is 50 $\mathrm{MeV} \equiv 1$ relativistic particle) and a logarithmic channel with a threshold $s_{o}=0.5 \mathrm{GeV}$. The signal from the fifth dynode of PM tube FEU-49 goes to a logarithmic channel (LC) where it is converted into a pulse whose length $\mathrm{t}$ is proportional to the logarithm of the amplitude of the signal [2].

There is a voluminous set of monitoring programs which allows us to examine the operation of each detector of the facility in any time interval and discard not properly working detectors.

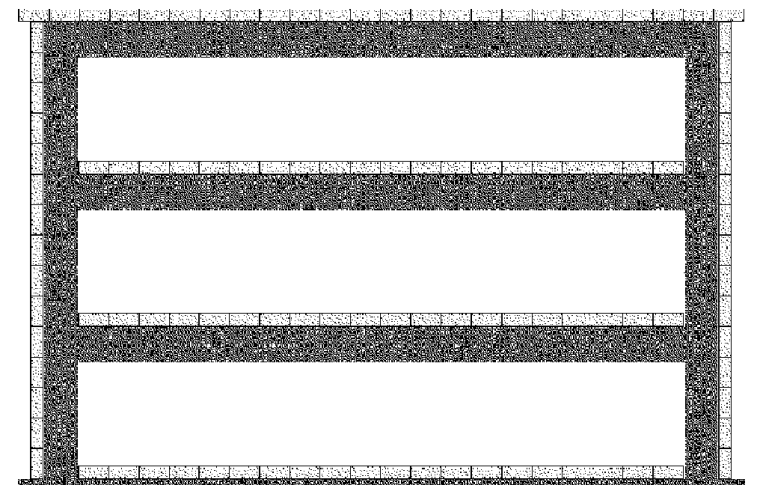

Fig. 1: General view of the Baksan underground scintillation telescope.

\section{THE METHOD OF NEUTRINO BURST DETECTION}

BUST consists of 3156 standard autonomous detectors. Each detector is filled with an organic scintillator $C_{n} H_{2 n+2},(n \simeq 9)$ and is viewed by one photomultiplier with a photocathode diameter of $15 \mathrm{~cm}$. The total scintillator mass is $330 \mathrm{t}$, and the mass enclosed in three lower horizontal layers (1200 standard detectors) is 130 tons. The neutrino signal from a supernova explosion is recorded with a help of a reaction

$$
\bar{\nu}_{e}+p \rightarrow n+e^{+}
$$

If the mean antineutrino energy is $E_{\nu_{e}}=12-15 \mathrm{MeV}$ [3], [4], [5] the pass of $e^{+}$(produced in reaction (1)) will be included, as a rule, in the volume of one detector. In such case the signal from a supernova explosion will appear as a series of events from singly triggered detectors (one and only one detector from 3156) during the neutrino burst. The search for a neutrino burst consists in recording of single events bunch within time interval of $\tau=20 \mathrm{~s}$.

If one assumes the distance from the star is $10 \mathrm{kpc}$ and the total energy irradiated in neutrinos is

$$
\varepsilon_{\text {tot }}=3 \times 10^{53} \mathrm{erg}
$$

the expected number of single events from reaction (1) (we assume the total energy of the $\bar{\nu}_{e}$ flux is equal to $\left.1 / 6 \times \varepsilon_{t o t}\right)$ will be

$$
N_{e v}^{H} \simeq 38 \times \eta_{1},
$$


where $\eta_{1}$ denotes the detection efficiency of $e^{+}$in reaction (1) and the symbol " $\mathrm{H}$ " indicates that the hydrogen is the target. $\eta_{1} \approx 0.7$ if the electron energy $E_{e}=10$ $\mathrm{MeV}$ and $\eta_{1}=0.9$ if $E_{e}=20 \mathrm{MeV}$.

Background events are radioactivity, ghost signals from detectors and cosmic ray muons if only one detector from 3156 hit. The total count rate from background events is $\mathrm{n}=0.02 \mathrm{~s}^{-1}$ in internal planes (three lower horizontal layers) and $1 s^{-1}$ in external ones. Therefore three lower horizontal layers are used as a target (the estimation (3) has been calculated for three internal planes).

Background events can imitate the expected signal $(\mathrm{k}$ single events within sliding time interval $\tau$ ) with a count rate

$$
p(k)=n \times \exp (-n \tau) \frac{(n \tau)^{k-1}}{(k-1) !}
$$

The treatment of experimental data (background events over a period $2001-2008 \mathrm{y} ; \mathrm{T}=236126 \mathrm{RUNs}$, RUN = $900 \mathrm{~s}$ ) is shown by squares in Fig.2 in comparison with the expected distribution according to the expression (4).

If the scenario of 2-stage collapse [6] is realized in Nature and the mean neutrino energy (during the first stage) is $\bar{E}_{\nu_{e}}=30-40 \mathrm{MeV}$, the following reactions begin to work:

$$
\begin{aligned}
& \nu_{i}+{ }^{12} \mathrm{C} \rightarrow{ }^{12} \mathrm{C}^{*}+\nu_{i}, \quad E_{t h}=15.1 \mathrm{MeV}, \\
& i=e, \mu, \tau, \\
& { }^{12} \mathrm{C}^{*} \rightarrow{ }^{12} \mathrm{C}+\gamma, \quad E_{\gamma}=15.1 \mathrm{MeV}
\end{aligned}
$$

and

$$
\begin{array}{ll}
\nu_{e}+{ }^{12} \mathrm{C} \rightarrow{ }^{12} \mathrm{~N}+e^{-}, & E_{t h}=17.34 \mathrm{MeV}, \\
{ }^{12} N \rightarrow{ }^{12} \mathrm{C}+e^{+}+\nu_{e}, & \tau=15.9 \mathrm{~ms},
\end{array}
$$

$\tau$ is a lifetime of the nucleus ${ }^{12} N$.

Reaction (5) allows to measure the total neutrino flux with the energy $E_{\nu}>15.1 \mathrm{MeV}$.

If the mean energy $\bar{E}_{\nu}=30 \mathrm{MeV}$ the expected number of events for reactions (5) and (6) can be estimated (under conditions (2)) by formulae

$$
\begin{aligned}
& N_{e v 2}^{C}=16 \times \eta_{2}\left(E_{\gamma}=15 \mathrm{MeV}\right), \\
& N_{e v 3}^{C}=30 \times \eta_{3}\left(E_{\nu}=30 \mathrm{MeV}\right),
\end{aligned}
$$

The radiation length for our scintillator is $47 \mathrm{~g} / \mathrm{cm}^{2}$ therefore $\eta_{2} \approx 0.2$. In reaction (6) BUST can detect both $e^{-}$with energy $E_{\nu}-17 \mathrm{MeV}$ and $e^{+}$if the energy deposition from these particles is greater $8 \mathrm{MeV}$. In the latter case, the reaction (6) will have the distinctive signature: two signals separated with $5-45 \mathrm{~ms}$ time interval (dead time of the BUST is $\simeq 1 \mathrm{~ms}$ ).

In reaction (6) the sum of energies $E_{e^{+}}+E_{\nu}$ is 17.3 $\mathrm{MeV}$ therefore $\eta_{3} \approx 0.5-0.7$.

The low part of the overlap between horizontal scintillation planes is the $8 \mathrm{~mm}$ iron layer. This can be used as the target in the reaction

$$
\nu_{e}+{ }^{56} \mathrm{Fe} \rightarrow{ }^{56} \mathrm{Co}^{*}+e^{-}, \quad E_{t h}=4.056 \mathrm{MeV} \text {, }
$$

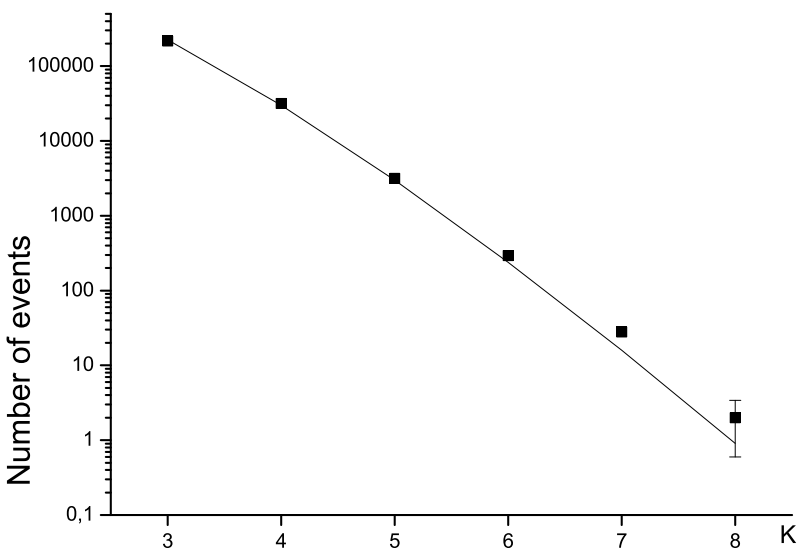

Fig. 2: The number of bunches with $k$ single events within time interval of $\tau=20 \mathrm{~s}$. Squares are experimental data, the curve is the expected number according to the expression (4).

(cobalt emerges in excited state).

Under conditions (2) the expected number of events from reaction (9) (neutrinos arrive from above) is

$$
N_{e v}^{F e}=6.3 \times \eta_{F e}(26 \mathrm{MeV}),
$$

$\eta_{F e}(26 \mathrm{MeV}) \approx 0.4$ is the detection efficiency of $e^{-}$ with the energy $26 \mathrm{MeV}$ produced into the $8 \mathrm{~mm}$ iron layer.

It should be noticed, if $\bar{E}_{\nu_{e}}=30-40 \mathrm{MeV}$ a noticeable percentage of neutrino reactions (9) will cause triggering two detectors.

Since 2001 all events are collected by the facility. Earlier only the events selected by physics programs (by means of corresponding electronic systems) were recorded. All events recording allows us to observe any events before and after single events.

\section{RESULTS}

So, if the scenario of 2-stage collapse [6] is realized in Nature the signal from collapse (the number of neutrino induced events) is increased $\approx 50 \%$.

The facility has been operating under the program of search for collapse neutrinos since the mid-1980 [7]. The observation time is $T=24.7$ years. Let $f_{\text {col }}$ be the mean frequency of collapses. The probability of collapse absence during the time interval $T$ is (according to the Poisson law) $\exp \left(-f_{\text {col }} T\right)$. An upper bound on the mean frequency of gravitational collapses in the Galaxy at $90 \%$ CL can be obtained with the help of the expression

$$
\exp \left(-f_{\text {col }} T\right)=0.1
$$

Thus

$$
f_{\text {col }}<0.093 y^{-1}, \quad 90 \% C L
$$

Acknowledgements. The work is supported by Russian Fund for Basic Research (grants no. 07-02-00162 
and 09-02-00434), Russian Academy of Sciences Basic Research Program "Neutrino Physics and Astrophysics" and State Program of Supporting Leading Scientific Schools (grant no. NSh-321.2008.2).

\section{REFERENCES}

[1] Alexeyev E.N., Alexeyenko V.V. et al. Proc. of 16 ICRC, Kyoto, 1979, v. 10, p. 276.

[2] Bakatanov V.N., Novoseltsev Yu.F. et al. Proc. of 17 ICRC, Paris, 1981, v.7, p. 67.

[3] Imshennik V.S., Nadezhin D.K., Itogi Nauki i Tehniki, ser. Astronomy v.21, p.63 (1982)

[4] Hillebrandt W., Hoflish P., Rep.Prog.Phys. v.52, p.1421 (1989)

[5] Imshennik V.S. ITEP Preprint 135-90 (1990)

[6] Imshennik V.S. Space Sci.Rev. v.74, p.325 (1995)

[7] Alexeyev E.N., Alexeyeva L.N. et al. JETP, v. 104, 2897 (1993) 